\title{
Technical Evolution of Medical Endoscopy
}

\author{
S. Gross, M. Kollenbrandt
}

\begin{abstract}
This paper gives a summary of the technical evolution of medical endoscopy. The first documented redirection of sunlight into the human body dates back to the 16th century. Rigid tubes with candle light were given a trial later on. Low light intensity forced the development of alternative light sources. Some of these experiments included burning chemical components. Electric lighting finally solved the problems of heat production and smoke. Flexible endoscopy increased the range of medical examinations as it allowed access to tight and angular body cavities. The first cameras for endoscopic applications made taking photos from inside the human body possible. Later on, digital video endoscopy made endoscopes easier to use and allowed multiple spectators to observe the endoscopic intervention. Swallowable capsules called pill-cams made endoscopic examinations of the small intestine possible. Modern technologies like narrow band imaging and fluorescence endoscopy increased the diagnostic significance of endoscopic images. Today, image processing is applied to decrease noise and enhance image quality. These enhancements have made medical endoscopy an invaluable tool in many diagnostic processes. In closing, an example is given of an interdisciplinary examination, which is taken from the archaeological field.
\end{abstract}

Keywords: Technical evolution of endoscopy, medical examination history, flexible endoscopy, rigid endoscopy, photo endoscopy, spectral endoscopy, capsule endoscopy, wireless endoscopy, image processing.

\section{Introduction}

Endoscopy is a vital part of medical diagnostic processes and an everyday tool in today's medical environments. The evolution of endoscopic technologies was driven by the desire to gain information on the patient's medical status from the optical appearance of body cavities. Data on the biological processes inside the body were hard to come by. Medical practitioners, engineers, and inventors participated in the endeavor to bring light into the body and guide it out again.

A review of the technical evolution of medical endoscopy is given in this paper. The first steps in the development, which are centered around rigid endoscopy are described in section 2. The advances in flexible endoscopy which led to an increased range in the human body are highlighted in section 3. The introduction of photographic documentation is the topic of section 4 . The focus of section 5 is on the adoption of video technology in endoscopy. In section 6 the use of spectral imaging in endoscopy is described. Swallowable capsules for endoscopic examination are discussed in section 7 . The increased significance of endoscopic images by means of image processing is the topic of section 8. An example of an interdisciplinary application of medical imaging technology is shown in section 9. Closing remarks are given in section 10 .

\section{Rigid endoscopy}

Giulio Cesare Aranzi (1530-1589), an Italian physician, was the first medical practitioner to direct sunlight into a body cavity. This medical examination took place in 1585. He used a flask of water to reflect light into his patient's nose [1]. There were several later descriptions of the use sunlight and its redirection into the human body. These examinations include some conducted by Waldenburg and Kussmaul (both in 1870).

The first technologically successful attempt to guide light into the human body was undertaken by Philipp Bozzini (1773-1808) [2]. He was an Italian physician who grew up in Germany. His apparatus called 'Lichtleiter' (German, translated to English: light conductor) was constructed from a metal casing which was designed to hold a candle [3]. A schematic drawing of the Lichtleiter can be seen in Fig. 1.
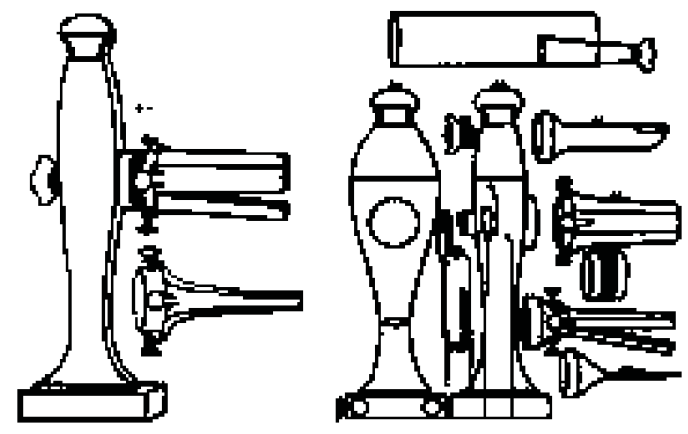

Fig. 1: Schematic drawing of Bozzini's Lichtleiter

On the one side of the casing Bozzini placed holes, to which he attached metal tubes. The tubes were used to guide the light into the human body. Tubes of different sizes were available for different body openings. He even invented a tube with a mirror to redirect the light to the vocal cords. On the other side of the casing was an opening for the observer to look into.

The apparatus was a revolutionary idea. The disadvantages were the heat and smoke created by the candle. Consequently, the invention did not gain wide acceptance among medical practitioners [4]. Unfortunately, Philipp Bozzini died of typhus at the age of 35 and did not live to anticipate the changes he inspired with his creation.

Antonin Jean Desormeaux (1815-1882) replaced the candle with a mixture of alcohol and turpentine to increase the illumination. However, this solution still created heat and sometimes smoke while burning. Furthermore, he used condenser lenses to concentrate the illumination on a single spot during the examinations. Desormeaux conducted the first successful operative endoscopic procedures in living patients, and is considered by many as the 'father of endoscopy' [6]. 
The lighting system was further improved by Julius Bruck (1840-1902), a dentist. He was the first to suggest inserting a light source into the human body. In 1867 he installed a platinum wire into a water reservoir and in this way created an easily applicable light source.

Max Nitze (1848-1906) was a general practitioner interested in medical examination of the urinary bladder. He was the first inventor who created an endoscope with the light source at its tip. After experimenting with Julius Bruck's platinum wire, he miniaturized Edison's filament globe and created the first cystoscope in 1877. The cystoscope contained several optical lenses used to guide the light through the tube and to the observer's eye. However, collaborating with several medical instrument makers he failed to claim the patent for his invention and a patent war between several parties erupted [7].

Johann von Mikulicz-Radecki (1850-1905) used Nitze's concept and introduced a mirror to create the angular field of view still found in many of today's endoscopic, devices [5]. He also added an air canal to his endoscope which enabled the examiner to inflate the body cavity under observation. This greatly increased the field of view and allowed the inspection of otherwise collapsed body cavities. His rigid gastroscope was $650 \mathrm{~mm}$ long and $13 \mathrm{~mm}$ in diameter.

A new type of light transmission was introduced by Fourestier in 1952. A rigid quartz rod just $1.5 \mathrm{~mm}$ in diameter was inserted into a $2 \mathrm{~mm}$ stainless steel tube. The quartz rod was later used in the development of the first highquality movie films.

\section{Flexible endoscopy}

The first recorded flexible esophagoscope was invented by Kelling in 1898. The lower third of this endoscope could be flexed up to an angle of $45^{\circ}$. The instrument maker who manufactured the endoscope was Albright. Both Kelling and Albright might be called pioneers of gastroscopy [2].

Schindler introduced an improved version of a semiflexible gastroscope in 1936. The flexible lower third was $12 \mathrm{~mm}$ in diameter and contained a spiral which hosted fixings for more than 48 lenses. The rigid part of the endoscope was $8.5 \mathrm{~mm}$ in diameter. The illumination was supplied by an electric globe. The maximum bending angle for the endoscope was $30^{\circ}$, as exceeding this angle caused the transmission of the image to fail. The system was an improvement over existing technologies but was still not flawless, as blind spots existed which could not be visualized.

\section{Photoendoscopy}

Maximilian Nitze (1848-1906) realized that creating suitable photos is of special importance in the medical field. Photos made sharing information with colleagues or creating records for later documentation possible. He invented a cystoscope which could hold glass plates with a light sensitive coating [3]. The plates could be moved into the light and an exposure time of 3-5 seconds was necessary to create a photograph.

Lange and Meltzing invented a small camera that was attached to a rubber tube and could be swallowed. Their idea was to create images from the inside of the esophagus without the need for an endoscope which could guide the light out of the body. The front part was still rigid and contained the camera, a film magazine for film rolls with 50 exposures, and an electric globe for illumination. The rubber tube hosted the electric wiring for the globe, the mechanical camera trigger, and an air channel for insufflation of the body cavities. The disadvantage of this design was that the examiner was not aware of the actual position of the camera or the field of view. Only after the images had been processed one could say whether the images were of any diagnostic value.

\section{Video endoscopy}

Conventional endoscopy had always caused a lot of restrictions for the investigator. Similar to using a microscope, the observer needed one eye to look along the optical axis of the endoscope. This was uncomfortable at best, and only a single person could observe the body cavities at a time. Taking images from the inside of the body was tedious, and was often done without knowing what would actually be on the photos. A remedy for this situation was video endoscopy.

The first reported video endoscopy was conducted by Soulas in France in 1956. Video equipment was still very large and heavy, as miniaturization had not been a focus. In fact, the easiest way to get video signals from the human body was to take the patient to a television studio. A rigid endoscope was fixed to a regular television camera, and video images were transmitted to a television monitor. These first steps were cumbersome, but with the development of better equipment video endoscopy became the standard method for medical examinations and interventions. However, the first endoscopic video images were black \& white only.

A team in Melbourne, Australia, miniaturized the camera in 1960. It was $45 \mathrm{~mm}$ wide and $120 \mathrm{~mm}$ long with a reduced weight of $350 \mathrm{~g}$. The camera could be attached to a regular endoscope and could transmit the images to a screen. However, the images were still black and white and a monochrome display was used. There was little enthusiasm in the clinical world.

The introduction of digital imaging with charge-coupled device (CCD) image sensors in 1985 was a breakthrough. The chips could be miniaturized and the whole imaging procedure could take place at the tip of the endoscope. The fiber bundles used to guide the light in optical systems could be replaced by wires. The flexibility of the endoscopes increased and the image quality rose. Now digital video data could be easily processed digitally, and noise filtering and image enhancement were built-in post processing steps of the endoscopic video units.

\section{Spectral endoscopy}

Technological advancements have not only increased the flexibility, the image quality, or the operational reach of endoscopes, but have also changed the kind of information that they can retrieve. Standard white light endoscopy is still a very important aspect of endoscopic examinations. However, spectral endoscopy has evolved and allows the detection or easy diagnosis of additional diseases. The use of various illumination techniques has enhanced the visibility of features that cannot be distinguished under white light. 
An example of this is Narrow-Band imaging (NBI) [8]. A filter wheel is moved in front of the white light source, which reduces the emitted light to two narrow bands. The blue light at $415 \mathrm{~nm}$ enhances the superficial capillary network, while the green light at $540 \mathrm{~nm}$ displays subepithelial vessels. The combination of the two light bands highlights the blood vessel pattern, which can be essential for certain diagnoses.

For example, in their 2007 paper Tischendorf et al. [9] presented a promising study on the diagnostic relevance of the blood vessel pattern in the differentiation between benign and possibly malignant colorectal polyps. The difference between a white light image and an NBI image can be seen in Fig. 2.
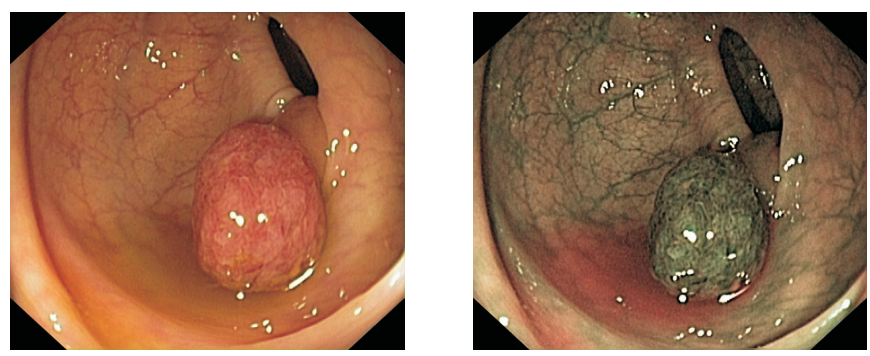

Fig. 2: Two images (white light on the left, Narrow-band imaging on the right) showing the same scene. One can easily see the difference. The blood vessel pattern is emphasized in the image on the right.

Another example for the diagnostic value of spectral imaging is fluorescence endoscopy. Since the 1960's urologists have investigated ways to label bladder tumors in vivo [10]. However, staining with tetracyclines, methylene blue, fluorescein or synthetic porphyrin compounds could not be established as standard procedures and they were abandoned. In 1995 an investigation was made of a fluorescing agent called ALA [11], which is injected into the patient's blood circuit. The agent is transported into all parts of the patient's body and accumulates in areas with high metabolic activity. During a consecutive bladder examination special lighting is used to activate the fluorescence, which then indicates any tumorous growth on the bladder wall. An example of the discriminative power of fluorescence endoscopy is highlighted in Fig. 3.
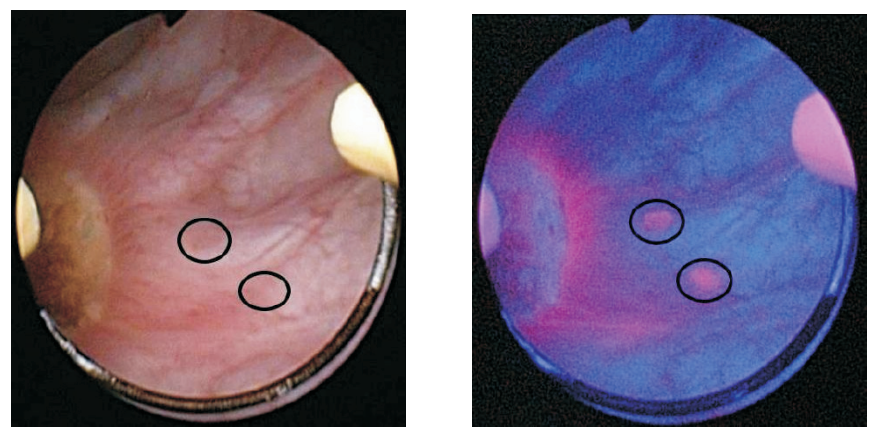

Fig. 3: The image on the left side shows a white light image from bladder endoscopy. There is no evidence of tumors. On the right side the same location is imaged with bladder fluorescence endoscopy. Two tumors can easily bespotted. The images give a clear example of the discriminative power of spectral imaging in endoscopy.

\section{Capsule endoscopy}

One of the recent developments in the field of endoscopy is capsule endoscopy, where a mini-camera in capsule form is given to the patient for swallowing. An Israeli physician, Gavriel Iddan, started developing a pill-sized camera in 1981. However, miniaturization and battery technology were not ready for his idea. However, in 2001 the American Food and Drug Administration approved a camera of the size of a pill $(26 \times 11 \mathrm{~mm})$ for endoscopic purposes. The pill contained a digital camera, its battery, control and transceiver electronics and was covered by a plastic coating. After the capsule had been swallowed, the camera took two images per second. During the eight hour procedure approximately 60000 images were taken. An image of a capsule endoscope can be seen in Fig. 4.

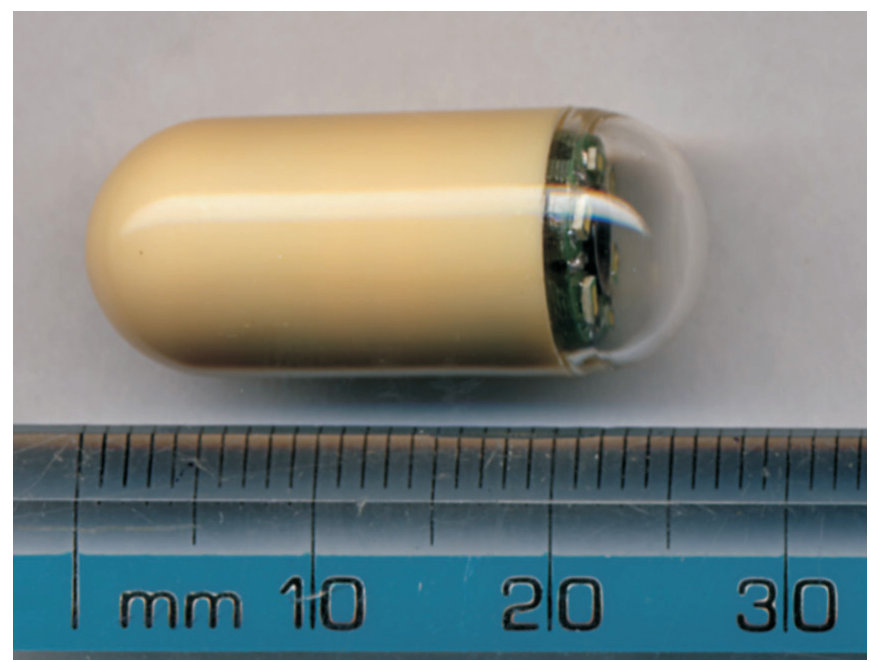

Fig. 4: Image of a capsule endoscope (pill-cam) with size reference

So-called pill-cams are primarily used to visualize diseases in the small intestine which is too long for an examination using traditional scopes. However, the number of images created during the long procedure forces medical staff to spend much time on the analysis. This can sometimes take up to two hours, which is longer than a standard colonoscopy or gastroscopy. Leading research in this field is now being done in the United States, Japan, and Germany.

\section{Computer-based image processing in medical endoscopy}

Image processing is a crucial part of the efforts to increase the quality and significance of medical images. Numerous filtering and image quality enhancement techniques have been introduced for various modalities and situations. A small sample selection is presented here.

In the endoscopic field a wide range of methods and ideas have been suggested. An example of quality enhancement for fluorescence endoscopy which is described in section 6 , is contrast enhancement [12]. Other efforts concentrate on correction of systematic errors introduced by endoscopic imaging equipment. To this end, Stehle et al. suggested a dynamic distortion correction for endoscopy systems with exchangeable 
optics [13]. A main focus of research on capsule endoscopes is on reducing the time humans need to review the data created during the procedure. Automatic video browsing and region of interest tagging, as well as content evaluation, are among the most common research projects [14, 15]. Mosaicking techniques have been adapted to create panoramic views from endoscopic images, which are otherwise locally restricted [16].

\section{Interdisciplinary applications}

While investigating on the historical evolution of endoscopic technology one recognizes that endoscopic equipment is used in a multitude of different application fields. These fields include security, science and criminal investigations. Endoscopes are also used in maintenance tasks for technical equipment like the engines of airplanes. Endoscopic technologies have fostered new ways of research, especially in interdisciplinary contexts. Disciplines like archeology and biology can often profit from research and developments in the medical field.

An excellent example of an archaeological application is the examination of Oetzi the Iceman [17]. The 5300 year old mummy from the neolithic age was found in a glacier in the Alps between Austria and Italy. An endoscopic examination of the stomach via the esophagus (gastroscopy) was performed amongst other medical examinations of the corpse. The contents of the stomach gave indications of the eating habits of human kind in the late stone age. Remains of whip-worms, which are parasites causing diarrhea and anemia, were also found during the analysis. An endoscopic examination of the colon (colonoscopy) substantiated this suspicion, as a large number of whip-worm eggs were found. This led to the conclusion that the Iceman had an infestation caused by the whip-worm parasite.

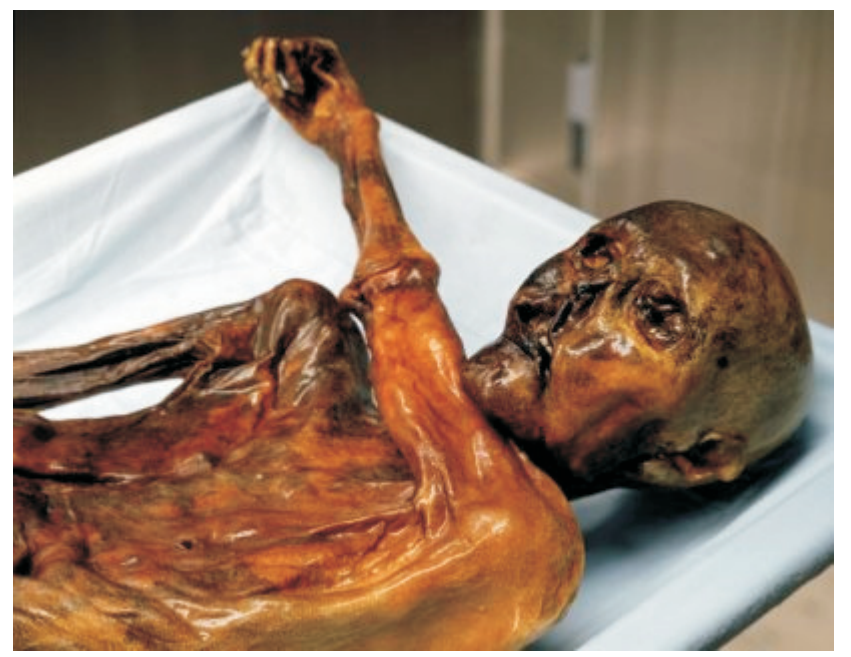

Fig. 5: Image of Oetzi the Iceman during examinations

\section{Conclusion}

Endoscopy is an integral part of many of today's medical diagnostic processes. Engineers have tried to extend the boundaries of endoscopic imaging ever since the first physicians used sun light to look into the human body. The pioneering years were spent trying to guide light into the body and back out again. While only inelastic tubes were used in those days, various light sources and mirroring techniques were invented. Rigid endoscopy is still used today for many investigations and surgical interventions. There were, however, parts of visceral cavities that could not be reached. To this end flexible endoscopy was invented to catch a glimpse of narrow and angulate body cavities like the colon. Medical practitioners gained additional insight into the human body. Photographic equipment for endoscopies gave the opportunity to take images from the inside of the human body. But this idea was soon replaced by video endoscopy, which made the clinical routine easier and gave multiple spectators the chance to follow interventions. Spectral imaging was introduced to increase the significance of endoscopic images and create new diagnostic methods for many disease patterns. But even with all these advances the small intestine was still out of reach for investigators, and capsule endoscopes were developed to close this diagnostic gap. Today computing power is exploited, e.g. to reduce noise in endoscopic images and support medical practitioners in their decisions. All these advancements make medical endoscopy a unique and important tool in the clinical routine. However, the benefits of endoscopic equipment and of the inventions made to increase its value in clinical environments are not restricted to the medical field.

\section{Acknowledgments}

The authors would like to thank Univ.-Prof. Dr. phil. Christine Roll, head of the Institute of History, RWTH Aachen University, and Univ.-Prof. Dr.-Ing. Til Aach, head of the Institute of Imaging \& Computer Vision, RWTH Aachen University, for their kind support and guidance.

\section{References}

[1] Aranzi, G. C.: Hippocratis librum de vulneribus captitis commentarius cum claudii porralii annotationibus marginalibus. Lugduni: Apud Ludovicum Cloquemin, 1580.

[2] Berci, G., Forde, K. A.: History of Endoscopy: what Lessons Have we Learned from the Past? In Surg Endosc, Vol. 14 (2000), No. 1, p. 5-15.

[3] Prevedello, D. M., Doglietto, F., Jane, J. A. Jr., Jagan Nathan, J., Han, J., Laws, E. R. Jr.: History of Endoscopic Skull Base Surgery: its Volution and Current Reality. In $J$ Neurosurg, Vol. 107 (2007), p. 206-213.

[4] Bozzini, P. H.: Lichtleiter, eine Erfindung zur Anschauung innerer Teile und Krankheiten. In J Prak Heilk, Vol. 24 (1806).

[5] Zajaczkowski, T.: Johann Anton von Mikulicz-Radecki (1850-1905) - a Pioneer of Gastroscopy and Modern Surgery: his Credit to Urology. In WorldJ Urol, Springer, Vol. 26 (2008), No. 1, p. 75-86.

[6] Lamaro, V. P.: Gynaecological Endoscopic Surgery Past, Present and Future. In St. Vincent's Clinic, Proceedings, Vol. 12 (2004), No. 1, p. 23-29.

[7] Nitze, M.: Zur Photographie der menschlichen Harnblase. In Med Wochenschr, Vol. 178 (1893), No. 2.

[8] Gono, K., Obi, T., Yamaguchi, M., Ohyama, N., Machida, H., Sano, Y., Yoshida, S., Hamamoto, Y., Endo, T.: Appearance of Enhanced Tissue Features in 
Narrow-Band Endoscopic Imaging. In Journal of Biomedical Optics, Vol. 9 (2004), No. 3, p. 568-577.

[9] Tischendorf, J. J. W., Wasmuth, H. E., Koch, A., Hecker, H., Trautwein, C., Winograd, R.: Value of Magnifying Chromoendoscopy and Narrow Band Imaging (NBI) in Classifying Colorectal Polyps: a Prospective Controlled Study. In Endoscopy, Vol. 39 (2007), No. 12, p. 1092-1096.

[10] Zaak, D., Karl, A., Knchel, R., Stepp, H., Hartmann, A., Reich, O., Bachmann, A., Siebels, M., Popken, G., Stief, C.: Diagnosis of Urothelial Carcinoma of the Bladder Using Fluorescence Endoscopy. In BJU Int., Vol. 96 (2005), No. 2, p. 217-222.

[11] Steinbach, P., Weingandt, H., Baumgartner, R., Kriegmair, M., Hofstdter, F., Knchel, R.: Cellular Fluorescence of the Endogeneous Photosensitizer Protoporphyrin IX Following Exposure to 5-Aminolevulinic Acid. In Photochem Photobiol, Vol. 62 (1995), p. 88795.

[12] Stehle, T., Behrens, A., Aach, T.: Enhancement of Visual Contrast in Fluorescence Endoscopy. In Proceedings of the IEEE International Conference on Multimedia and Expo, June 2008, p. 537-540.

[13] Stehle, T., Hennes, M., Gross, S., Behrens, A., Wulff, J., Aach, T.: Dynamic Distortion Correction for Endoscopy Systems with Exchangeable Optics. In Bildverarbeitung für die Medizin 2009, Springer, Berlin (Germany), 2009.

[14] Bourbakis, N.: Detecting Abnormal Patterns in wce Images. In Fifth IEEE Symposium on Bioinformatics and Bioengineering 2005, BIBE 2005, October 2005, p. 232-238.
[15] Kodogiannis, V.: Computer-Aided Diagnosis in Clinical Endoscopy Using Neuro-Fuzzy Systems. In Proceedings IEEE International Conference on Fuzzy Systems 2004, Vol. 3 (2004), p. 1425-1429.

[16] Behrens, A.: An Image Mosaicing Algorithm for Bladder Fluorescence Endoscopy. In Proceedings of the $12^{\text {th }}$ International Student Conference on Electrical Engineering POSTER 2008, Prague (Czech Republic), May 2008.

[17] Spindler, K.: The Man in the Ice: the Preserved Body of a Neolithic Man Reveals the Secrets of the Stone Age. London (Great Britain): Weidenfeld \& Nicolson, January 1994.

Sebastian Gross

e-mail: sebastian.gross@lfb.rwth-aachen.de

Institute of Imaging \& Computer Vision

RWTH Aachen University

D-52056 Aachen, Germany

Medical Department III

RWTH Aachen University Hospital

Pauwelsstr. 30, D-52074 Aachen, Germany

Maren Kollenbrandt

e-mail: maren.kollenbrandt@rwth-aachen

Institute of History

RWTH Aachen University

D-52056 Aachen, Germany 